\title{
Basic result on type II DM self-motions of planar Stewart Gough platforms
}

\author{
G. Nawratil \\ Institute of Discrete Mathematics and Geometry, \\ Vienna University of Technology, Austria, \\ e-mail: nawratil@geometrie.tuwien.ac.at
}

\begin{abstract}
In a recent publication [10] the author showed that self-motions of general planar Stewart Gough platforms can be classified into two so-called Darboux Mannheim (DM) types (I and II). Moreover, in [10] the author was able to compute the set of equations yielding a type II DM selfmotion explicitly. Based on these equations we present a basic result for this class of self-motions.
\end{abstract}

Key words: Self-motion, Stewart Gough platform, Borel Bricard problem

\section{Introduction}

The geometry of a planar Stewart Gough (SG) platform is given by the six base anchor points $\mathrm{M}_{i}$ with coordinates $\mathbf{M}_{i}:=\left(A_{i}, B_{i}, 0\right)^{T}$ with respect to the fixed system $\Sigma_{0}$ and by the six platform anchor points $\mathrm{m}_{i}$ with coordinates $\mathbf{m}_{i}:=\left(a_{i}, b_{i}, 0\right)^{T}$ with respect to the moving system $\Sigma$. By using Study parameters $\left(e_{0}: \ldots: e_{3}: f_{0}: \ldots: f_{3}\right)$ to parametrize Euclidean displacements, the coordinates $\mathbf{m}_{i}^{\prime}$ of the platform anchor points with respect to $\Sigma_{0}$ can be written as $K \mathbf{m}_{i}^{\prime}=\mathbf{R} \mathbf{m}_{i}+\left(t_{1}, t_{2}, t_{3}\right)^{T}$ with

$$
\begin{aligned}
& t_{1}=2\left(e_{0} f_{1}-e_{1} f_{0}+e_{2} f_{3}-e_{3} f_{2}\right), \quad t_{2}=2\left(e_{0} f_{2}-e_{2} f_{0}+e_{3} f_{1}-e_{1} f_{3}\right) \\
& t_{3}=2\left(e_{0} f_{3}-e_{3} f_{0}+e_{1} f_{2}-e_{2} f_{1}\right), \quad K=e_{0}^{2}+e_{1}^{2}+e_{2}^{2}+e_{3}^{2} \neq 0 \quad \text { and } \\
& \mathbf{R}=\left(r_{i j}\right)=\left(\begin{array}{ccc}
e_{0}^{2}+e_{1}^{2}-e_{2}^{2}-e_{3}^{2} & 2\left(e_{1} e_{2}-e_{0} e_{3}\right) & 2\left(e_{1} e_{3}+e_{0} e_{2}\right) \\
2\left(e_{1} e_{2}+e_{0} e_{3}\right) & e_{0}^{2}-e_{1}^{2}+e_{2}^{2}-e_{3}^{2} & 2\left(e_{2} e_{3}-e_{0} e_{1}\right) \\
2\left(e_{1} e_{3}-e_{0} e_{2}\right) & 2\left(e_{2} e_{3}+e_{0} e_{1}\right) & e_{0}^{2}-e_{1}^{2}-e_{2}^{2}+e_{3}^{2}
\end{array}\right)
\end{aligned}
$$

Now all points of $P_{\mathbb{R}}^{7}$ which are located on the so-called Study quadric $\Psi: \sum_{i=0}^{3} e_{i} f_{i}=$ 0 , correspond to an Euclidean displacement, with exception of the subspace $e_{0}=$ $\ldots=e_{3}=0$ of $\Psi$, as these points cannot fulfill the normalizing condition $K=1$.

If the geometry of the manipulator is given as well as the six leg lengths, then the SG platform is in general rigid, but it can even be the case that the manipulator can perform an $n$-parametric motion ( $n>0)$, which is called self-motion. Note that such motions are also solutions to the famous Borel Bricard problem (cf. $[1,3,4,11])$. 


\section{Types of self-motions}

In section 2 and 3 we give a very short review of the results and ideas stated in [10], where also more details and examples can be found.

It is known that architecturally singular SG platforms, which are well studied, possess self-motions in each pose. Therefore, we are only interested in the computation of self-motions of non-architecturally singular SG platforms. A detailed review of self-motions of this type was given by the author in [10].

Moreover, it is known that if a planar SG platform with anchor points $m_{1}, \ldots, M_{6}$ is not architecturally singular, then at least a one-parametric set of legs exists, which can be attached to the given manipulator without changing the forward kinematics $[5,9]$ and the singularity set [2] of the manipulator. Moreover, it was shown that in general the base anchor points $\mathrm{M}_{i}$ as well as the corresponding platform anchor points $\mathrm{m}_{i}$ are located on planar cubic curves $C$ and $c$, respectively.

Assumption 1. We assume that there exist such cubics $\mathrm{C}$ and $\mathrm{C}$ (which can also be reducible) in the Euclidean domain of the platform and the base, respectively.

We consider the complex projective extension $P_{\mathbb{C}}^{3}$ of the Euclidean 3-space with $\left(a_{i}, b_{i}, 0\right) \mapsto\left(w_{i}: x_{i}: y_{i}: 0\right),\left(A_{i}, B_{i}, 0\right) \mapsto\left(W_{i}: X_{i}: Y_{i}: 0\right)$ and $w_{i}, x_{i}, y_{i}, W_{i}, X_{i}, Y_{i} \in \mathbb{C}$. Note that ideal points are characterized by $w_{i}=0$ and $W_{i}=0$, respectively.

Moreover, we consider the correspondence between the points of $\mathrm{C}$ and $\mathrm{c}$, which is determined by the geometry of the manipulator $m_{1}, \ldots, M_{6}$ (cf. $\left.[2,5,9]\right)$. As this correspondence has not to be a bijection, a point $\in P_{\mathbb{C}}^{3}$ of $\mathrm{c}$ resp. $\mathrm{C}$ is in general mapped to a non-empty set of points $\in P_{\mathbb{C}}^{3}$ of $C$ resp. c. We denote this set by the term corresponding location and indicate this fact by the usage of brackets \{\} .

In $P_{\mathbb{C}}^{3}$ the cubic $C$ has three ideal points $U_{1}, U_{2}, U_{3}$, where at least one of these points $\left(\right.$ e.g. $\left.U_{1}\right)$ is real. The remaining points $U_{2}$ and $U_{3}$ are real or conjugate complex. Then we compute the corresponding locations $\left\{u_{1}\right\},\left\{u_{2}\right\},\left\{u_{3}\right\}$ of $c\left(\Rightarrow\left\{u_{1}\right\}\right.$ contains real points). We denote the ideal points of $c$ by $u_{4}, u_{5}, u_{6}$, where again one (e.g. $u_{4}$ ) has to be real. The remaining points $u_{5}$ and $u_{6}$ are again real or conjugate complex. Then we compute the corresponding locations $\left\{\mathrm{U}_{4}\right\},\left\{\mathrm{U}_{5}\right\},\left\{\mathrm{U}_{6}\right\}$ of $\mathrm{C}(\Rightarrow$ $\left\{\mathrm{U}_{4}\right\}$ contains real points).

Assumption 2. For guaranteeing a general case, we assume that each of the corresponding locations $\left\{\mathrm{u}_{1}\right\},\left\{\mathrm{u}_{2}\right\},\left\{\mathrm{u}_{3}\right\},\left\{\mathrm{U}_{4}\right\},\left\{\mathrm{U}_{5}\right\},\left\{\mathrm{U}_{6}\right\}$ consists of a single point. Moreover, we assume that no 4 collinear platform anchor points $\mathrm{u}_{j}$ or base anchor points $\mathrm{U}_{j}(j=1, \ldots, 6)$ exist.

Under consideration of Assumption 1 and 2, following theorem was proven [10]:

Theorem 1. The resulting manipulator $\mathrm{u}_{1}, \ldots, \mathrm{U}_{6}$ is architecturally singular.

Moreover, it was proven in [10] that there only exist type I and type II Darboux Mannheim (DM) self-motions, where the definition of types reads as follows:

Definition 1. Assume $\mathscr{M}$ is a one-parametric self-motion of a non-architecturally singular SG platform $\mathrm{m}_{1}, \ldots, \mathrm{M}_{6}$. Then $\mathscr{M}$ is of the type $n \mathrm{DM}$ if the corresponding architecturally singular manipulator $\mathrm{u}_{1}, \ldots, \mathrm{U}_{6}$ has an $n$-parametric self-motion. 


\section{Computation of type II DM self-motions}

The only examples of type II DM self-motions known to the author are those constructed by Karger in [7,8], which are characterized by $e_{0}=0$.

The computation of type II DM self-motions in [10] was based on Darboux and Mannheim constraints, which are repeated next. With this approach it seems for the first time possible to give a complete classification of type II DM self-motions:

Darboux constraint: The constraint that the platform anchor point $\mathrm{u}_{i}(i=1,2,3)$ moves in a plane of the fixed system orthogonal to the direction of the ideal point $\mathrm{U}_{i}$ can be written as (cf. [10])

$$
\Omega_{i}: \bar{X}_{i}\left(a_{i} r_{11}+b_{i} r_{12}+t_{1}\right)+\bar{Y}_{i}\left(a_{i} r_{21}+b_{i} r_{22}+t_{2}\right)+L_{i} K=0
$$

with $X_{i}, Y_{i}, a_{i}, b_{i}, L_{i} \in \mathbb{C}$. This is a homogeneous quadratic equation in the Study parameters where $\bar{X}_{i}$ and $\bar{Y}_{i}$ denote the conjugate complex of $X_{i}$ and $Y_{i}$, respectively.

Mannheim constraint: The constraint that the plane orthogonal to $\mathrm{u}_{i}(i=4,5,6)$ through the platform point $\left(g_{i}, h_{i}, 0\right)$ slides through the point $\mathrm{U}_{i}$ of the fixed system can be written as (cf. [10])

$$
\begin{aligned}
& \Pi_{i}: \bar{x}_{i}\left[A_{i} r_{11}+B_{i} r_{21}-g_{i} K-2\left(e_{0} f_{1}-e_{1} f_{0}-e_{2} f_{3}+e_{3} f_{2}\right)\right]+ \\
& \bar{y}_{i}\left[A_{i} r_{12}+B_{i} r_{22}-h_{i} K-2\left(e_{0} f_{2}+e_{1} f_{3}-e_{2} f_{0}-e_{3} f_{1}\right)\right]=0,
\end{aligned}
$$

with $x_{i}, y_{i}, A_{i}, B_{i}, g_{i}, h_{i} \in \mathbb{C}$. This is again a homogeneous quadratic equation in the Study parameters where $\bar{x}_{i}$ and $\bar{y}_{i}$ denote the conjugate complex of $x_{i}$ and $y_{i}$.

The content of the following lemma was also proven in [10]:

Lemma 1. Without loss of generality (w.l.o.g.) we can assume that the variety of the two-parametric self-motion of $\mathrm{u}_{1}, \ldots, \mathrm{U}_{6}$ is spanned by $\Psi, \Omega_{1}, \Omega_{2}, \Omega_{3}, \Pi_{4}, \Pi_{5}$. Moreover, we can choose following special coordinate systems in $\Sigma_{0}$ and $\Sigma$ w.l.o.g.: $X_{1}=Y_{2}=Y_{3}=x_{4}=y_{5}=1, a_{1}=b_{1}=y_{4}=A_{4}=B_{4}=Y_{1}=h_{4}=g_{5}=0$ and $X_{2}\left(X_{2}-X_{3}\right) x_{5} \neq 0$.

We solve the linear system of equations $\Psi, \Omega_{1}, \Omega_{2}, \Pi_{4}$ for $f_{0}, \ldots, f_{3}$ and plug the obtained expressions in the remaining two equations. ${ }^{1}$ This yields in general two homogeneous polynomials $\Omega[40]$ and $\Pi[96]$ in the Euler parameters of degree 2 and 4, respectively. The number in the square brackets gives the number of terms.

Finally, we compute the resultant of $\Omega$ and $\Pi$ with respect to one of the Euler parameters. Here we choose ${ }^{2} e_{0}$. This yields a homogeneous polynomial $\Gamma[117652]$ of degree 8 in $e_{1}, e_{2}, e_{3}$. In the following we denote the coefficients of $e_{1}^{i}, e_{2}^{j}, e_{3}^{k}$ of $\Gamma$ by $\Gamma_{i j k}$. We get a set $\mathscr{E}$ of 24 equations $\Gamma_{i j k}=0$ in the 14 unknowns $a_{2}, b_{2}, a_{3}, b_{3}, A_{5}, B_{5}, X_{2}, X_{3}, x_{5}, L_{1}, L_{2}, L_{3}, g_{4}, h_{5}$.

Moreover, it should be noted that we denote the coefficients of $e_{0}^{i} e_{1}^{j}, e_{2}^{k}, e_{3}^{l}$ of $\Omega$ and $\Pi$ by $\Omega_{i j k l}$ and $\Pi_{i j k l}$, respectively.

\footnotetext{
${ }^{1}$ For $e_{0} e_{2}-e_{1} e_{3} \neq 0$ this can be done w.l.o.g., as this factor belongs to the denominator of $f_{i}$.

${ }^{2}$ Therefore we are looking for a common factor of $\Omega$ and $\Pi$, which depends on $e_{0}$.
} 


\section{The basic result}

An important step in direction of a complete classification of type II DM selfmotions is done by the basic result given in Theorem 2. As preparatory work for the formulation of this theorem we have to define the following two special cases: It can easily be seen, that $\Omega$ does not depend on $e_{0}$ and $e_{3}$ (upper signs) or $e_{1}$ and $e_{2}$ (lower signs) if the following three equations are fulfilled:

$$
\begin{aligned}
& L_{1}\left(\bar{X}_{2}-\bar{X}_{3}\right)-L_{2}+L_{3}=0, \\
& a_{2}\left(\bar{X}_{2}-\bar{X}_{3}\right) \pm \bar{X}_{3}\left(\bar{X}_{2} b_{2}-\bar{X}_{3} b_{3}\right) \pm b_{2} \mp b_{3}=0, \\
& a_{3}\left(\bar{X}_{2}-\bar{X}_{3}\right) \pm \bar{X}_{2}\left(\bar{X}_{2} b_{2}-\bar{X}_{3} b_{3}\right) \pm b_{2} \mp b_{3}=0 .
\end{aligned}
$$

Theorem 2. With exception of the above mentioned two special cases, the corresponding manipulator $\mathrm{u}_{1}, \ldots, \mathrm{U}_{6}$ of a planar $S G$ platform (fulfilling Assumptions 1, 2 and Lemma 1) with a type II DM self-motion, has to have further 3 collinear anchor points in the base or in the platform beside the points $\mathrm{U}_{1}, \mathrm{U}_{2}, \mathrm{U}_{3}$ and $\mathrm{u}_{4}, \mathrm{u}_{5}, \mathrm{u}_{6}$.

The proof is done by contradiction, i.e. we stop the case study if 3 anchor points beside $\mathrm{U}_{1}, \mathrm{U}_{2}, \mathrm{U}_{3}$ and $\mathrm{u}_{4}, \mathrm{u}_{5}, \mathrm{u}_{6}$ are collinear or if we get one of the 2 special cases.

Proof for the general case $\Omega_{2000} \Pi_{3000} \neq 0$

We assume $\Omega_{200} \Pi_{3000} \neq 0$, as only those solutions of $\mathscr{E}$ correspond to type II selfmotions, which do not cause a vanishing of the coefficient of the highest power of $e_{0}$ in $\Omega$ and $\Pi$, respectively.

$\Gamma_{800}$ can only vanish without contradiction (w.c.) for $L_{1}=g_{4}$ or if $F=\bar{X}_{2}\left(L_{1}-\right.$ $\left.a_{2}\right)-\bar{X}_{3}\left(L_{1}-a_{3}\right)-L_{2}+L_{3}+b_{2}-b_{3}$ is fulfilled identically. We distinguish 3 parts:

Part [A] Assuming $L_{1} \neq g_{4}$ : Now $F=0$ has to hold. W.l.o.g. we express $L_{1}$ from $F=0$. Then $\Gamma_{710}=0$ implies $a_{2}=a_{3}-\bar{X}_{2} b_{2}+\bar{X}_{3} b_{3}$. Now $\Gamma_{620}$ cannot vanish w.c..

Part [B] $L_{1}=g_{4}$ and $F=0$ : We express $L_{1}$ from $F=0$. W.l.o.g. we can compute $h_{5}$ from the only non-contradicting factor of $\Gamma_{602}$. Now $\Gamma_{530}$ can vanish w.c. for:

1. $L_{3}=\bar{X}_{3}\left(L_{2}-b_{2}\right) / \bar{X}_{2}+\bar{X}_{3}\left(a_{2}-a_{3}\right)+b_{3}$ : W.l.o.g. we can express $A_{5}$ from the only non-contradicting factor of $\Gamma_{422}$. Again we distinguish two cases:

a. $\bar{X}_{2} b_{2}-\bar{X}_{3} b_{3}+a_{2}-a_{3} \neq 0$ : Now $\Gamma_{350}$ has only one non-contradicting factor, which can be solved for $L_{2}$ w.l.o.g.. Then we can solve the only noncontradicting factor of $\Gamma_{314}$ for $\bar{x}_{5}$ w.l.o.g.. Now the resultant of the only noncontradicting factors of $\Gamma_{206}$ and $\Gamma_{242}$ with respect to $B_{5}$ cannot vanish w.c..

b. $a_{3}=\bar{X}_{2} b_{2}-\bar{X}_{3} b_{3}+a_{2}$ : Then $\Gamma_{314}=0$ implies $L_{2}=2 \bar{X}_{2}^{2} b_{2}+\bar{X}_{2} a_{2}+b_{2}$.

i. $\bar{X}_{3}\left(\bar{X}_{2} b_{2}-\bar{X}_{3} b_{3}\right)+a_{2}\left(\bar{X}_{3}-\bar{X}_{2}\right)+\bar{x}_{5}^{2}\left(b_{3}-b_{2}\right) \neq 0$ : Under this assumption we can express $B_{5}$ from the only non-contradicting factor of $\Gamma_{242}$. Then $\Gamma_{224}$ can only vanish w.c. for $\bar{X}_{i}=-\bar{x}_{5}$ with $i=2$ or $i=3$. As for $\bar{x}_{5} b_{j}+\bar{X}_{j} b_{i}=0$ with $i \neq j$ and $i, j \in\{2,3\}$ the expression $\Gamma_{080}$ cannot vanish w.c., we can assume $\bar{x}_{5} b_{j}+\bar{X}_{j} b_{i} \neq 0$. Under this assumption we can compute $a_{2}$ from $\Gamma_{080}=0$ w.l.o.g.. Then the linear-combination $\Gamma_{044}-\Gamma_{026}-\Gamma_{062}$ equals $b_{2}^{2} b_{3}^{2}\left(\bar{X}_{j}+\bar{x}_{5}\right)^{2}\left(b_{2}-b_{3}\right)$, a contradiction. 
ii. $\bar{X}_{3}\left(\bar{X}_{2} b_{2}-\bar{X}_{3} b_{3}\right)+a_{2}\left(\bar{X}_{3}-\bar{X}_{2}\right)+\bar{x}_{5}^{2}\left(b_{3}-b_{2}\right)=0$ : W.l.o.g. we can solve this equation for $a_{2}$. Then $\Gamma_{242}$ can only vanish w.c. for $\bar{X}_{i}=-\bar{x}_{5}$ and $\Gamma_{080}=0$ implies $\bar{X}_{j}=\bar{x}_{5}$ with $i \neq j$ and $i, j \in\{2,3\}$. Now $\bar{x}_{5}^{2} \Gamma_{206}-\Gamma_{026}$ equals $b_{i} b_{j}^{2} B_{5} \bar{x}_{5}^{6}\left(b_{2}-b_{3}\right)$, a contradiction.

2. $a_{2}=\bar{X}_{3} b_{3}-\bar{X}_{2} b_{2}+a_{3}$ and $\bar{X}_{2} \bar{X}_{3}\left(a_{2}-a_{3}\right)+\bar{X}_{2}\left(b_{3}-L_{3}\right)-\bar{X}_{3}\left(b_{2}-L_{2}\right) \neq 0$ : In this case $\Gamma_{440}=0$ already yields the contradiction.

Part [C] Assuming $F \neq 0$ : Now $L_{1}=g_{4}$ has to hold. Then $\Gamma_{080}$ factors into $G[8] H[18]^{2}$. We distinguish 3 cases:

1. $G[8]=0$ : W.1.o.g. we can express $L_{1}$ from $G[8]=0$. Now $\Gamma_{170}$ can only vanish w.c. for $\left(\bar{X}_{3} b_{3}-\bar{X}_{2} b_{2}-a_{2}+a_{3}\right) V[18]=0$ :

a. $a_{2}=\bar{X}_{3} b_{3}-\bar{X}_{2} b_{2}+a_{3}$ : We can solve the only non-contradicting factor of $\Gamma_{620}$ for $h_{5}$. Now we can express $L_{3}$ from the only non-contradicting factor of $\Gamma_{602}$. Moreover, we can compute $A_{5}$ from the only non-contradicting factor of $\Gamma_{260}$. Then we can solve the only non-contradicting factor of $\Gamma_{062}$ for $L_{2}$.

i. $\bar{x}_{5}^{2}\left(b_{2}-b_{3}\right)+2 \bar{x}_{5}\left(\bar{X}_{2} b_{2}-\bar{X}_{3} b_{3}\right)+a_{3}\left(\bar{X}_{3}-\bar{X}_{2}\right)+\bar{X}_{2}\left(\bar{X}_{2} b_{2}-\bar{X}_{3} b_{3}\right) \neq 0$ : Now we can compute $B_{5}$ from the only non-contradicting factor of $\Gamma_{404}$ w.l.o.g.. We distinguish two cases:

$\star \bar{X}_{2} b_{3}-\bar{X}_{3} b_{2} \neq 0$ : Under this assumption we can express $a_{3}$ from the only non-contradicting factor of $\Gamma_{026}$. Then $\Gamma_{206}$ cannot vanish w.c..

$\star \bar{X}_{2}=\bar{X}_{3} b_{2} / b_{3}$ : Now $\Gamma_{026}=0$ implies $b_{2}=-b_{3}$. Finally $\Gamma_{206}=0$ yields the contradiction.

ii. $\bar{x}_{5}^{2}\left(b_{2}-b_{3}\right)+2 \bar{x}_{5}\left(\bar{X}_{2} b_{2}-\bar{X}_{3} b_{3}\right)+a_{3}\left(\bar{X}_{3}-\bar{X}_{2}\right)+\bar{X}_{2}\left(\bar{X}_{2} b_{2}-\bar{X}_{3} b_{3}\right)=0$ :

W.l.o.g. we can solve this equation for $a_{3}$. Then $\Gamma_{206}$ can only vanish w.c.

for the following two cases:

$\star \bar{X}_{3}=-\bar{x}_{5}$ : Now $\Gamma_{404}=0$ implies $b_{2}=-b_{3}$ and from $\Gamma_{422}=0$ we get $B_{5}=-b_{3}$. Then $\Gamma_{440}-\Gamma_{242}=0$ yields the contradiction.

$\star \bar{X}_{2}=-\bar{x}_{5}: \Gamma_{404}=0$ implies $b_{2}=-b_{3}$ and $\Gamma_{422}$ cannot vanish w.c.

b. $V[18]=0, \bar{X}_{3} b_{3}-\bar{X}_{2} b_{2}-a_{2}+a_{3} \neq 0$ : W.l.o.g. we can solve this equation for $A_{5}$. We can solve the only non-contradicting factor of $\Gamma_{620}$ for $h_{5}$. Then we can express $L_{3}$ from the only non-contradicting factor of $\Gamma_{602}$. Moreover, we can solve the only non-contradicting factor of $\Gamma_{062}$ for $L_{2}$.

i. $\bar{x}_{5}^{2}\left(b_{3}-b_{2}\right)+\bar{x}_{5}\left(a_{2}-a_{3}+\bar{X}_{3} b_{3}-\bar{X}_{2} b_{2}\right)+\bar{X}_{2} a_{2}-\bar{X}_{3} a_{3} \neq 0$ : Under this assumption we can solve $\Gamma_{404}=0$ for $B_{5}$. Then $\Gamma_{026}=0$ implies $a_{2}=$ $\bar{X}_{3} a_{3} b_{2} /\left(\bar{X}_{2} b_{3}\right)$ and $\Gamma_{206}=0$ yields the contradiction.

ii. $\bar{x}_{5}^{2}\left(b_{3}-b_{2}\right)+\bar{x}_{5}\left(a_{2}-a_{3}+\bar{X}_{3} b_{3}-\bar{X}_{2} b_{2}\right)+\bar{X}_{2} a_{2}-\bar{X}_{3} a_{3}=0$ :

$\star a_{2}-\bar{x}_{5} b_{2} \neq 0$ : In this case we can express $\bar{X}_{2}$ from the above equation. Now $\Gamma_{206}=0$ implies $a_{3}=\bar{x}_{5} b_{3}$. From $\Gamma_{404}=0$ we get $a_{2}=-\bar{X}_{3} b_{2}$ and $\Gamma_{422}=0$ yields $B_{5}=b_{3}$. Then $\Gamma_{440}-\Gamma_{242}$ cannot vanish w.c..

$\star a_{2}=\bar{x}_{5} b_{2}:$ Now $\Gamma_{026}=0$ implies $b_{3}=\bar{X}_{3} a_{3} /\left(\bar{X}_{2} \bar{x}_{5}\right)$ and from $\Gamma_{422}=0$ we get $\bar{X}_{3}=-\bar{x}_{5}$. Then $\Gamma_{440}-\Gamma_{242}=0$ yields the contradiction.

2. $H[18]=0, G[8] \neq 0$ and $\bar{X}_{2} a_{2}-\bar{X}_{3} a_{3} \neq 0$ : Under this assumption we can compute $h_{5}$ from $H[18]=0$. Then we can express $B_{5}$ from the only non-contradicting 
factor of $\Gamma_{620}$. Moreover, from the only non-contradicting factor of $\Gamma_{602}$ we can compute $L_{2}$. Then we consider the only non-contradicting factor $I[14]$ of $\Gamma_{260}$.

a. $\bar{x}_{5}^{2}\left(b_{2}-b_{3}\right)+\bar{x}_{5}\left(\bar{X}_{3} b_{3}-\bar{X}_{2} b_{2}-a_{2}+a_{3}\right)+\bar{X}_{2} a_{2}-\bar{X}_{3} a_{3} \neq 0$ : Under this assumption we can express $A_{5}$ from $I[14]=0$.

i. $a_{3}-\bar{x}_{5} b_{3} \neq 0$ : Under this assumption we can express $L_{3}$ from the only non-contradicting factor of $\Gamma_{062}$. Then $\Gamma_{044}=0$ implies $a_{2}=\bar{X}_{2} b_{2}-$ $\bar{X}_{3} b_{3}+a_{3}$. Then the resultant of $\Gamma_{404}$ and $\Gamma_{422}$ with respect to $\bar{x}_{5}$ can only vanish w.c. for $J[12]\left(\bar{X}_{2} b_{3}-a_{3}\right)\left(\bar{X}_{3} b_{2}+\bar{X}_{3} b_{3}-\bar{X}_{2} b_{2}-a_{3}\right)=0$ :

$\star J[12]=0$ : W.l.o.g. we can solve this equation for $a_{3}$. Then $\Gamma_{404}=0$ implies $\bar{X}_{3}=\bar{x}_{5}$. Now $\Gamma_{026}$ cannot vanish w.c..

$\star a_{3}=\bar{X}_{3} b_{2}+\bar{X}_{3} b_{3}-\bar{X}_{2} b_{2}$ : Then $\Gamma_{404}$ cannot vanish w.c..

$\star a_{3}=\bar{X}_{2} b_{3}$ : Now $\Gamma_{404}=0$ implies $\bar{X}_{3}=\bar{x}_{5}$ and $\Gamma_{206}$ cannot vanish w.c..

ii. $a_{3}=\bar{x}_{5} b_{3}$ : From $\Gamma_{062}=0$ we get $L_{1}=0$. Then $\Gamma_{404}=0$ implies $a_{2}=$ $\bar{X}_{2} b_{2}-\bar{X}_{3} b_{2}+\bar{x}_{5} b_{2}$. Now $\Gamma_{422}$ cannot vanish w.c..

b. $\bar{x}_{5}^{2}\left(b_{2}-b_{3}\right)+\bar{x}_{5}\left(\bar{X}_{3} b_{3}-\bar{X}_{2} b_{2}-a_{2}+a_{3}\right)+\bar{X}_{2} a_{2}-\bar{X}_{3} a_{3}=0$ :

i. $\bar{X}_{3} \neq \bar{x}_{5}$ : Under this assumption we can express $a_{3}$ from the above equation. Then $I=0$ implies $\bar{X}_{2}=\bar{x}_{5}$. Now we can solve the only non-contradicting factor of $\Gamma_{404}$ for $A_{5}$ w.l.o.g.. Then $\Gamma_{026}=0$ implies $a_{2}=\bar{X}_{3} b_{2}$. Now the difference of the only non-contradicting factors of $\Gamma_{062}$ and $\Gamma_{422}$ can only vanish w.c. for:

$\star \bar{X}_{3}=1 / \bar{x}_{5}$ : Then we can solve $\Gamma_{062}=0$ for $L_{3}$ w.l.o.g.. Finally $\Gamma_{044}=0$ yields the contradiction.

$\star L_{1}=4 b_{2} b_{3}\left(\bar{x}_{5}-\bar{X}_{3}\right) /\left(b_{2}-b_{3}\right)$ : Now $\Gamma_{062}$ cannot vanish w.c..

ii. $\bar{X}_{3}=\bar{x}_{5}$ : The equation of item $\mathrm{b}$ can only vanish w.c. for $a_{2}=\bar{x}_{5} b_{2}$. Then we can express $A_{5}$ w.l.o.g. from the only non-contradicting factor of $\Gamma_{404}$. Now $\Gamma_{026}=0$ implies $a_{3}=\bar{X}_{2} b_{3}$. Then the difference of the only noncontradicting factors of $\Gamma_{062}$ and $\Gamma_{422}$ can only vanish w.c. for:

$\star \bar{X}_{2}=1 / \bar{x}_{5}$ : Now we can solve $\Gamma_{062}=0$ for $L_{3}$ w.l.o.g.. Then $\Gamma_{044}=0$ implies $b_{2}=-b_{3}$. Finally $\Gamma_{026}=0$ yields the contradiction.

$\star L_{1}=4 b_{2} b_{3}\left(\bar{X}_{2}-\bar{x}_{5}\right) /\left(b_{2}-b_{3}\right)$ : Now $\Gamma_{062}$ cannot vanish w.c..

3. $H[18]=0, G[8] \neq 0$ and $a_{2}=\bar{X}_{3} a_{3} / \bar{X}_{2}$ : Now $H=0$ implies $A_{5}=-\bar{X}_{3} a_{3} / \bar{x}_{5}$. W.l.o.g. we can express $h_{5}$ from the only non-contradicting factor of $\Gamma_{620}$. Moreover, the only non-contradicting factor of $\Gamma_{602}$ can be solved w.l.o.g. for $L_{2}$. Then we consider the only non-contradicting factor $E[12]$ of $\Gamma_{260}$.

a. $\bar{X}_{2} \bar{x}_{5}\left(b_{3}-b_{2}\right)-\bar{X}_{2}\left(\bar{X}_{3} b_{3}-\bar{X}_{2} b_{2}\right)-a_{3}\left(\bar{X}_{2}-\bar{X}_{3}\right) \neq 0$ : Under this assumption we can solve $E[12]=0$ for $B_{5}$. Then $\Gamma_{062}=0$ implies $a_{3}=\bar{x}_{5} b_{3}$. Now we can express $\bar{x}_{5}$ w.l.o.g. from the only non-contradicting factor of $\Gamma_{404}$. Then $\Gamma_{026}=0$ implies $\bar{X}_{3}=0$. Finally $\Gamma_{044}=0$ yields the contradiction.

b. $\bar{X}_{2} \bar{X}_{5}\left(b_{3}-b_{2}\right)-\bar{X}_{2}\left(\bar{X}_{3} b_{3}-\bar{X}_{2} b_{2}\right)-a_{3}\left(\bar{X}_{2}-\bar{X}_{3}\right)=0$ : W.l.o.g. we can express $a_{3}$ from this equation. Then $E$ can only vanish w.c. for:

i. $\bar{X}_{3}=\bar{x}_{5}$ : Now $\Gamma_{062}=0$ already yields the contradiction.

ii. $\bar{X}_{2}=\bar{x}_{5}$ : Now $\Gamma_{062}=0$ implies $B_{5}=-b_{3}$. Then we can solve the only non-contradicting factor of $\Gamma_{404}$ for $\bar{x}_{5} . \Gamma_{026}=0$ yields the contradiction. 
Proof for the special case $\Omega_{2000} \Pi_{3000}=0$

If we set $e_{i}$ equal to zero for any $i \in\{0, \ldots, 3\}$, then $\Omega$ and $\Pi$ have to be fulfilled identically. It can be seen immediately that the conditions implied by $\Omega=0$ already yield a contradiction. Therefore we can assume $e_{0} e_{1} e_{2} e_{3} \neq 0$ w.l.o.g. for this section of the proof.

Part [A] $\Omega_{2000}=0, \Omega_{1000} \Pi_{3000} \neq 0$ : From $\Omega_{2000}=0$ we can express $L_{1}$ w.l.o.g.. Moreover, we can compute $e_{0}$ from $\Omega=0$ and plug the resulting expression into $\Pi$ which yields in the numerator a homogeneous polynomial $\Gamma[10058]$ of degree 7 in $e_{1}, e_{2}, e_{3}$. From $\Gamma_{700}=0$ we can compute $g_{4}$. Then $\Gamma_{610}=0$ yields the contradiction.

Part [B] $\Omega_{2000}=\Pi_{3000}=0, \Omega_{1000} \Pi_{2000} \neq 0$ : Again we express $L_{1}$ from $\Omega_{2000}=0$. It can be seen immediately from $\Omega=0$ that all coefficients of $\Pi_{3000}=0$ with respect to the remaining Study parameters have to vanish in order to get no contradiction. Therefore we can compute $g_{4}$ and $h_{5}$ from $\Pi_{3100}=0$ and $\Pi_{3010}=0$, respectively. We solve $\Omega=0$ for $e_{0}$ and plug it into $\Pi$ which yields in the numerator a homogeneous polynomial $\Gamma[1666]$ of degree 5 in $e_{1}, e_{2}, e_{3}$.

W.l.o.g. we can compute $L_{3}$ from $\Gamma_{500}=0$. Then we can solve the only noncontradicting factor of $\Gamma_{410}$ for $L_{2}$. Moreover, the only non-contradicting factor of $\Gamma_{320}$ can be solved for $A_{5}$. Now $\Gamma_{302}=0$ has only one non-contradicting factor which can be solved for $\bar{x}_{5}$. Then the difference of the only non-contradicting factors of $\Gamma_{230}$ and $\Gamma_{104}$ can only vanish w.c. for $a_{2}=\bar{X}_{3} a_{3} b_{2} /\left(\bar{X}_{2} b_{3}\right)$.

1. $\bar{X}_{2}^{2} b_{3}^{2}\left(\bar{X}_{2} B_{5}-a_{3}\right)+\bar{X}_{3} a_{3}\left(\bar{X}_{2} b_{3}^{2}-B_{5} a_{3}\right) \neq 0$ : Now we can express $b_{2}$ from the only non-contradicting factor of $\Gamma_{230}$. Then $\Gamma_{014}$ cannot vanish w.c..

2. $\bar{X}_{2}^{2} b_{3}^{2}\left(\bar{X}_{2} B_{5}-a_{3}\right)+\bar{X}_{3} a_{3}\left(\bar{X}_{2} b_{3}^{2}-B_{5} a_{3}\right)=0$ :

a. $a_{3} B_{5}-\bar{X}_{2} b_{3}^{2} \neq 0$ : Under this assumption we can compute $\bar{X}_{3}$ from the above equation. Now $\Gamma_{230}=0$ implies $a_{3}=\bar{X}_{2} B_{5}$. Then $\Gamma_{014}$ cannot vanish w.c..

b. $a_{3}=\bar{X}_{2} b_{3}^{2} / B_{5}$ : Now the equation of item 2 can only vanish w.c. for $B_{5}= \pm b_{3}$. In both cases $\Gamma_{212}=0$ yields the contradiction.

Part [C] $\Omega_{2000}=\Pi_{3000}=\Pi_{2000}=0, \Omega_{1000} \Pi_{1000} \neq 0$ : Again we express $L_{1}$ from $\Omega_{2000}=0$. It can be seen immediately from $\Omega=0$ that all coefficients of $\Pi_{i 000}=$ 0 (for $i=2,3$ ) with respect to the remaining Study parameters have to vanish in order to get no contradiction. Therefore we compute $g_{4}$ and $h_{5}$ from $\Pi_{3100}=0$ and $\Pi_{3010}=0$, respectively. Moreover, we can solve $\Pi_{2101}=0$ and $\Pi_{2011}=0$ for $L_{3}$ and $L_{2}$ w.l.o.g.. We solve $\Omega=0$ for $e_{0}$ and plug it into $\Pi$ which yields in the numerator a homogeneous polynomial $\Gamma[191]$ of degree 5 in $e_{1}, e_{2}, e_{3}$.

Now $\Gamma_{410}=0$ implies $b_{2}=-B_{5}$. Then we can solve the only non-contradicting factor of $\Gamma_{320}$ for $A_{5}$ w.l.o.g.. Now $\Gamma_{230}=0$ implies $a_{2}=-B_{5} \bar{x}_{5}$. Then we can solve the only non-contradicting factor of $\Gamma_{302}$ for $a_{3}$ w.l.o.g.. We get $\bar{X}_{3}=-\bar{x}_{5}$ from $\Gamma_{104}=0$. Finally $\Gamma_{212}=0$ yields the contradiction.

Part [D] $\Pi_{3000}=0, \Omega_{2000} \Pi_{2000} \neq 0$ : It can be seen immediately from $\Omega=0$ that all coefficients of $\Pi_{3000}=0$ with respect to the remaining Study parameters have to vanish in order to get no contradiction. Therefore we can express $L_{1}$ and $h_{5}$ from $\Pi_{3100}=0$ and $\Pi_{3010}=0$, respectively. Then we compute the resultant of $\Omega[40]$ 
and $\Pi$ [44] with respect to $e_{0}$ which yields a homogeneous polynomial $\Gamma[15153]$ of degree 8 in $e_{1}, e_{2}, e_{3} . \Gamma_{080}$ can only vanish w.c. in the following 2 cases:

1. $L_{2}=g_{4}\left(\bar{X}_{2}-\bar{X}_{3}\right)+L_{3}+\bar{X}_{2} a_{2}-\bar{X}_{3} a_{3}-b_{2}+b_{3}$ : Then we can solve $\Gamma_{602}=0$ for $L_{3}$ w.l.o.g.. Now $\Gamma_{620}$ can only vanish w.c. in the following 2 cases:

a. $B_{5}=-b_{2}$ : Due to $\Gamma_{170}=0$ we have to distinguish again 2 cases:

i. $a_{2}=-A_{5} \bar{x}_{5} / \bar{X}_{2}$ : Then $\Gamma_{440}$ can only vanish w.c. for:

$\star \bar{X}_{2}=\bar{x}_{5}$ : Then $\Gamma_{062}=0$ implies $g_{4}=0$ and from $\Gamma_{026}=0$ we get $A_{5}=$ $-\bar{x}_{5} b_{2}$. Now $\Gamma_{242}=0$ implies $b_{3}=\bar{X}_{3} a_{3} / \bar{x}_{5}^{2}$. Finally $\Gamma_{422}=0$ yields the contradiction.

$\star A_{5}=-\bar{x}_{5} b_{2}$ : Again $\Gamma_{062}=0$ implies $g_{4}=0$. Moreover, we get $b_{3}=$ $\bar{X}_{3} a_{3} /\left(\bar{X}_{2} \bar{x}_{5}\right)$ from $\Gamma_{242}=0$ and $\bar{X}_{3}=-\bar{x}_{5}$ from $\Gamma_{206}=0$. Finally $\Gamma_{422}=0$ yields the contradiction.

$\star b_{2}=b_{3}-\bar{X}_{3} a_{3}-A_{5} \bar{x}_{5}$ : Again $\Gamma_{062}=0$ yields $g_{4}=0$. Then $\Gamma_{026}=0$ implies $\bar{X}_{2}=-\bar{x}_{5}$. We can express $b_{3}$ from the only non-contradicting factor of $\Gamma_{350}=0$. Then $\Gamma_{206}$ can only vanish w.c. for $S T=0$ with

$$
\begin{aligned}
& S=A_{5} \bar{x}_{5}\left(\bar{x}_{5}^{2}+1\right)+\bar{X}_{3} a_{3}\left(\bar{x}_{5}^{2}-1\right)-2 \bar{x}_{5} a_{3}, \\
& T=A_{5} \bar{x}_{5}\left(\bar{X}_{3}^{2}-1\right)+\bar{X}_{3} a_{3}\left(\bar{X}_{3}^{2}+1\right)-2 \bar{X}_{3} A_{5} .
\end{aligned}
$$

$\alpha) S=0$ : As for $\bar{x}_{5}= \pm i$ the equation $S=0$ cannot vanish w.c. we can assume w.l.o.g. $1+\bar{x}_{5}^{2} \neq 0$. Now we can express $A_{5}$ from $S=0$. Then $\Gamma_{224}=0$ implies $\bar{X}_{3}=1 / \bar{x}_{5}$. Finally $\Gamma_{242}=0$ yields the contradiction. ק) $T=0, \bar{x}_{5}\left(\bar{X}_{3}^{2}-1\right)-2 \bar{X}_{3} \neq 0$ : Under this assumption we can express $A_{5}$ from $T=0$. Then $\Gamma_{224}=0$ implies $\bar{X}_{3}=1 / \bar{x}_{5}$. Now $\Gamma_{242}=0$ yields the contradiction.

$\gamma) T=0, \bar{x}_{5}\left(\bar{X}_{3}^{2}-1\right)-2 \bar{X}_{3}=0$ : W.l.o.g. we can express $\bar{x}_{5}$ from the last equation. Then $T=0$ implies $a_{3}=0$. Now $\Gamma_{224}$ cannot vanish w.c..

ii. $a_{2}=a_{3}+\bar{X}_{3} b_{3}-\bar{X}_{2} b_{2}, \bar{X}_{2} a_{2}+A_{5} \bar{x}_{5} \neq 0$ : Then $\Gamma_{260}$ cannot vanish w.c.. b. $b_{2}=\bar{X}_{2} a_{2}-\bar{X}_{3} a_{3}+b_{3}, B_{5} \neq-b_{2}$ : Now $\Gamma_{530}$ cannot vanish w.c..

2. $a_{2}=-A_{5} \bar{x}_{5} / \bar{X}_{2}, g_{4}\left(\bar{X}_{2}-\bar{X}_{3}\right)-L_{2}+L_{3}+\bar{X}_{2} a_{2}-\bar{X}_{3} a_{3}-b_{2}+b_{3} \neq 0$ : W.l.o.g. we can express $b_{2}$ from the only non-contradicting factor of $\Gamma_{260}$. Then $\Gamma_{440}$ can only vanish w.c. for:

a. $\bar{X}_{2}=\bar{x}_{5}$ : Then $\Gamma_{602}=0$ implies an expression for $L_{3}$. Now $\Gamma_{404}$ can only vanish w.c in the following 2 cases:

i. $a_{3}=\bar{X}_{3} b_{3}+B_{5} \bar{x}_{5}-A_{5}$ : We distinguish again 2 cases:

$\star \bar{X}_{3} B_{5}+\bar{x}_{5} b_{3} \neq 0$ : Under this assumption we can compute $A_{5}$ from the only non-contradicting factor of $\Gamma_{206}$. Then we can solve the only noncontradicting factor of $\Gamma_{062}$ for $g_{4}$ w.l.o.g.. Now $\Gamma_{242}=0$ implies $\bar{X}_{3}=$ $-\bar{x}_{5}$ and $\Gamma_{422}=0$ yields the contradiction.

$\star \bar{X}_{3}=-\bar{x}_{5} b_{3} / B_{5}$ : Now $\Gamma_{206}=0$ implies $B_{5}=b_{3}$ and from $\Gamma_{062}=0$ we get an expression for $g_{4}$. Then we get $A_{5}=\bar{x}_{5} b_{3}$ from $\Gamma_{026}=0$ and $\Gamma_{422}=0$ yields the contradiction. 
ii. $L_{2}=\bar{x}_{5}\left(A_{5}+g_{4}\right)-B_{5}, \bar{X}_{3} b_{3}+B_{5} \bar{x}_{5}-A_{5}-a_{3} \neq 0$ : Then $\Gamma_{422}=0$ can be solved w.l.o.g. for $g_{4}$. From the only non-contradicting factor of $\Gamma_{314}$ we can express $a_{3}$ w.l.o.g.. We distinguish 2 cases:

$\star \bar{X}_{3} B_{5}+\bar{x}_{5} b_{3} \neq 0$ : Under this assumption we can solve $\Gamma_{242}=0$ for $A_{5}$. Then $\Gamma_{206}=0$ implies $\bar{X}_{3}=0$. Finally $\Gamma_{062}=0$ yields the contradiction. $\star \bar{X}_{3}=-\bar{x}_{5} b_{3} / B_{5}$ : Now $\Gamma_{242}=0$ cannot vanish w.c..

b. $A_{5}=\bar{X}_{2} B_{5}, \bar{X}_{2} \neq \bar{x}_{5}$ : W.l.o.g. we can solve $\Gamma_{602}=0$ for $L_{2}$. Then $\Gamma_{062}$ can only vanish w.c. for:

i. $g_{4}=0$ : Then $\Gamma_{422}=0$ implies $a_{3}=b_{3}\left(\bar{X}_{2}-\bar{X}_{3}+\bar{x}_{5}\right)$. Now $\Gamma_{242}=0$ yields $\bar{X}_{3}=\bar{x}_{5}$. Finally $\Gamma_{224}$ cannot vanish w.c.

ii. $B_{5}=-\bar{X}_{3} a_{3} /\left(\bar{X}_{2} \bar{x}_{5}\right), g_{4} \neq 0$ : Now the only non-contradicting factor of $\Gamma_{044}$ can be solved for $b_{3}$. Then $\Gamma_{026}=0$ yields the contradiction.

Part [E] $\Pi_{3000}=\Pi_{2000}=0, \Omega_{2000} \Pi_{1000} \neq 0$ : It can be seen immediately from $\Omega=0$ that all coefficients of $\Pi_{i 000}=0$ (for $i=2,3$ ) with respect to the remaining Study parameters have to vanish in order to get no contradiction. Therefore we can express $L_{1}$ and $h_{5}$ from $\Pi_{3100}=0$ and $\Pi_{3010}=0$, respectively. Moreover, we can compute $L_{2}$ and $g_{4}$ from $\Pi_{2101}=0$ and $\Pi_{2011}=0$, respectively. Then we solve $\Pi=0$ for $e_{0}$ and plug it into $\Omega$ which yields in the numerator a homogeneous polynomial $\Gamma[2408]$ of degree 8 in $e_{1}, e_{2}, e_{3}$.

Now $\Gamma_{602}$ can only vanish w.c. for $B_{5}=b_{2}$. Then we can solve the only noncontradicting factor of $\Gamma_{620}$ for $L_{3}$. Then $\Gamma_{530}=0$ implies $a_{2}=\bar{X}_{3} b_{3}-\bar{X}_{2} b_{2}+a_{3}$. Finally $\Gamma_{440}$ cannot vanish w.c..

Part [F] $\Omega_{2000}=\Omega_{1000}=0$ : W.l.o.g. we can express $L_{1}$ and $a_{2}$ from $\Omega_{2000}=0$ and $\Omega_{1001}=0$, respectively. As $\Omega_{0002}$ cannot vanish w.c. we proceed as follows:

1. $\Pi_{0003} \neq 0$ : Now we compute the resultant of $\Omega$ and $\Pi$ with respect to $e_{3}$ which yields a homogeneous polynomial $\Gamma[87839]$ of degree 8 in $e_{0}, e_{1}, e_{2}$. In the following we denote the coefficients of $e_{1}^{i}, e_{2}^{j}, e_{0}^{k}$ of $\Gamma$ by $\Gamma_{i j k}$. Now $\Gamma_{080}$ equals $\left(b_{2}-b_{3}\right) C[4] D[10]$.

a. $C[4]=0$ : W.1.o.g. we can express $a_{3}$ from $C=0$. Moreover, we can solve the only non-contradicting factor of $\Gamma_{800}$ for $A_{5}$ w.l.o.g.. Then we can compute $L_{2}$ from the only non-contradicting factor of $\Gamma_{170}$. Now $\Gamma_{206}=0$ implies $g_{4}=0$ and from $\Gamma_{404}=0$ we get $h_{5}=B_{5}-b_{3}-L_{3}$. Moreover, we can express $L_{3}$ from the only non-contradicting factor of $\Gamma_{062}$. Then $\Gamma_{026}=0$ implies $\bar{X}_{3}=0$ and finally $\Gamma_{620}=0$ yields the contradiction.

b. $D[10]=0, C[4] \neq 0$ : W.l.o.g. we can solve $D=0$ for $L_{2}$. Then $\Gamma_{206}=0$ implies $g_{4}=0$. Now we can solve the only non-contradicting factor of $\Gamma_{026}$ for $h_{5}$. Then $\Gamma_{404}=0$ implies $L_{3}=-b_{3}-\bar{X}_{3} a_{3}$. W.l.o.g. we can solve the only non-contradicting factor of $\Gamma_{602}$ for $a_{3}$. Now $\Gamma_{044}=0$ yields $A_{5}=B_{5} \bar{x}_{5}$. The difference of the only non-contradicting factors of $\Gamma_{260}$ and $\Gamma_{062}$ implies $\bar{X}_{2}=-\bar{X}_{3}$. Then $\Gamma_{260}$ can only vanish w.c. for:

i. $\bar{X}_{3}=\bar{x}_{5}: \Gamma_{620}=0$ implies $B_{5}=b_{3}$ and $\Gamma_{242}=0$ yields the contradiction. ii. $\bar{X}_{3}=-\bar{x}_{5}$ : Then $\Gamma_{620}=0$ already yields the contradiction. 
2. $\Pi_{0003}=0, \Pi_{0002} \neq 0$ : It can be seen immediately from $\Omega=0$ that all coefficients of $\Pi_{0003}=0$ with respect to the remaining Study parameters have to vanish in order to get no contradiction. Therefore we can express $h_{5}$ and $L_{2}$ from $\Pi_{0103}=0$ and $\Pi_{0013}=0$, respectively. Now we compute the resultant of $\Omega$ and $\Pi$ with respect to $e_{3}$ which yields a homogeneous polynomial $\Gamma$ [7821] of degree 8 in $e_{0}, e_{1}, e_{2}$. In the following we denote the coefficients of $e_{1}^{i}, e_{2}^{j}, e_{0}^{k}$ of $\Gamma$ by $\Gamma_{i j k}$. W.l.o.g. we can solve the only non-contradicting factor of $\Gamma_{800}$ for $a_{3}$. Moreover, we can compute $b_{2}$ from the only non-contradicting factor of $\Gamma_{620}$ w.l.o.g.. Then $\Gamma_{206}=0$ implies $g_{4}=0$. Now $\Gamma_{602}=0$ yields $A_{5}=B_{5} \bar{X}_{2}$. Then we solve the only non-contradicting factor of $\Gamma_{026}$ for $L_{3}$.

a. $\bar{X}_{3} B_{5}-\bar{X}_{2} b_{3} \neq 0$ : Under this assumption we can express $\bar{x}_{5}$ from the only non-contradicting factor of $\Gamma_{062}$. Then $\Gamma_{224}=0$ implies $\bar{X}_{2}=-\bar{X}_{3}$. Finally $\Gamma_{242}=0$ yields the contradiction.

b. $b_{3}=\bar{X}_{3} B_{5} / \bar{X}_{2}$ : Then $\Gamma_{062}=0$ implies $\bar{X}_{2}=-\bar{X}_{3}$. From $\Gamma_{224}=0$ we get $\bar{X}_{3}=-\bar{x}_{5}$. Finally $\Gamma_{242}=0$ yields the contradiction.

3. $\Pi_{0003}=\Pi_{0002}=0, \Pi_{0001} \neq 0$ : It can be seen immediately from $\Omega=0$ that all coefficients of $\Pi_{000 i}=0$ (for $i=2,3$ ) with respect to the remaining Study parameters have to vanish in order to get no contradiction. Therefore we can express $h_{5}$ and $L_{2}$ from $\Pi_{0103}=0$ and $\Pi_{0013}=0$, respectively. Moreover, we can compute $g_{4}$ and $L_{3}$ from $\Pi_{1102}=0$ and $\Pi_{1012}=0$, respectively. Then we solve $\Pi$ for $e_{3}$ and plug it into $\Omega$ which yields in the numerator a homogeneous polynomial $\Gamma[1766]$ of degree 8 in $e_{0}, e_{1}, e_{2}$. In the following we denote the coefficients of $e_{1}^{i}, e_{2}^{j}, e_{0}^{k}$ of $\Gamma$ by $\Gamma_{i j k}$.

W.l.o.g. we can solve the only non-contradicting factor of $\Gamma_{800}$ for $a_{3}$. Moreover, we can compute $b_{2}$ from the only non-contradicting factor of $\Gamma_{620}$ w.l.o.g.. Now $\Gamma_{206}=0$ yields $A_{5}=B_{5} \bar{X}_{2}$. Finally $\Gamma_{062}=0$ yields the contradiction.

4. $\Pi_{0003}=\Pi_{0002}=\Pi_{0001}=0, \Pi_{0300} \neq 0$ : It can be seen immediately from $\Omega=0$ that all coefficients of $\Pi_{000 i}=0$ (for $i=1,2,3$ ) with respect to the remaining Study parameters have to vanish in order to get no contradiction. Therefore we can express $h_{5}$ and $L_{2}$ from $\Pi_{0103}=0$ and $\Pi_{0013}=0$, respectively. Moreover, we can compute $g_{4}$ and $L_{3}$ from $\Pi_{1102}=0$ and $\Pi_{1012}=0$, respectively. $\Pi_{0121}=0$ implies $B_{5}=b_{2}$. From $\Pi_{2011}=0$ we get $A_{5}=a_{3}+\bar{x}_{5} b_{2}-\bar{X}_{3} b_{3}$. Now $\Pi_{0211}$ can only vanish w.c. for $\bar{X}_{2}=\bar{x}_{5}$.

As $\Omega_{0200}$ cannot vanish w.c. and due to our assumption $\Pi_{0300} \neq 0$ we can compute the resultant of $\Omega$ and $\Pi$ with respect to $e_{1}$ which yields a homogeneous polynomial $\Gamma[1013]$ of degree 6 in $e_{0}, e_{2}, e_{3}$. Now the coefficient of $e_{3}^{6}$ of $\Gamma$ cannot vanish w.c..

5. $\Pi_{0003}=\Pi_{0002}=\Pi_{0001}=\Pi_{0300}=0$ : Now we proceed analogously to the first and second paragraph of the last case but with the extra condition $\Pi_{0300}=0$ which implies $a_{3}=\bar{X}_{3} b_{3}$. Now $\Pi_{0200}$ and $\Omega_{0200}$ cannot vanish w.c. and therefore we can compute the resultant of $\Omega$ and $\Pi$ with respect to $e_{1}$ which yields a homogeneous polynomial $\Gamma[87]$ of degree 6 in $e_{0}, e_{2}, e_{3}$. Again the coefficient of $e_{3}^{6}$ of $\Gamma$ cannot vanish w.c.. 
Part [G] $\Pi_{3000}=\Pi_{2000}=\Pi_{1000}=0$ : In contrast to $\Pi_{1000}=0$, it can be seen immediately from $\Omega=0$ that all coefficients of $\Pi_{i 000}=0$ (for $i=2,3$ ) with respect to the remaining Study parameters have to vanish in order to get no contradiction. Therefore we can solve $\Pi_{3100}=0$ for $L_{1}, \Pi_{3010}=0$ for $h_{5}, \Pi_{2101}=0$ for $L_{2}$ and $\Pi_{2011}=0$ for $g_{4}$ w.l.o.g..

Part [G1] $\Pi_{1000}=0$ does not vanish identically for all $e_{1}, e_{2}, e_{3}$ : If the coefficient $Z$ of $e_{3}^{2}$ of $\Pi_{1000}$ vanishes, then $\Pi_{1000}=0$ only depends on $e_{1}, e_{2}$ and this already yields together with $\Omega=0$ the contradiction. Therefore we can assume w.l.o.g. $Z \neq 0$. Now we have to distinguish the following cases:

1. $\Omega_{0002} \Pi_{0003} \neq 0$ : We can compute the resultant of $\Pi_{1000}$ and $\Omega$ resp. $\Pi$ with respect to $e_{3}$ w.l.o.g. which yields $R_{\Omega}$ and $R_{\Pi}$, respectively. Now $R_{\Omega}$ and $R_{\Pi}$ have to vanish independently of $e_{0}, e_{1}, e_{2}$, whereby $R_{\Pi}$ spits up into $e_{2} P[8] Q[38]^{2}$. It can easily be seen that the coefficients of the quadratic homogeneous polynomial $P=0$ in the unknowns $e_{1}, e_{2}$ cannot vanish w.c.. Therefore we set $Q=0$ which is a quartic polynomial in $e_{1}, e_{2}$. We denote the coefficients of $e_{1}^{i} e_{2}^{j}$ of $Q$ by $Q_{i j}$. Now $Q_{04}$ can only vanish w.c. for:

a. $b_{2}=-B_{5}: Q_{40}=0$ implies an expression for $a_{2}$ and $Q_{31}$ cannot vanish w.c..

b. $A_{5}=a_{2}+B_{5} \bar{x}_{5}-\bar{X}_{2} b_{2}$ : Then $Q_{40}$ can vanish w.c. for:

i. $b_{2}=B_{5}$ : Then $Q_{31}=0$ implies an expression for $a_{2}$. Finally $Q_{13}$ cannot vanish w.c..

ii. $a_{2}=\bar{X}_{2} b_{2}$ : Now $Q_{31}$ can only vanish w.c. for $\bar{x}_{5}= \pm \bar{X}_{2}$. As for $\bar{x}_{5}=-\bar{X}_{2}$ the expression $Q_{22}$ cannot vanish w.c. we set $\bar{x}_{5}=\bar{X}_{2}$. Then $Q$ is fulfilled identically. Now it is not difficult to verify that the coefficients of $R_{\Omega}$ cannot vanish w.c. (proof is left to the reader).

2. $\Pi_{0003}=0, \Omega_{0002} \neq 0$ : W.l.o.g. we can compute $B_{5}$ from $\Pi_{0103}=0$.

a. $\bar{X}_{2} \neq \bar{x}_{5}$ : Under this assumption we can express $b_{2}$ from $\Pi_{0013}=0$. Now it can easily be seen that $\Pi_{0002}$ cannot vanish w.c.. Therefore we can compute the resultant of $\Pi_{1000}$ and $\Omega$ resp. $\Pi$ with respect to $e_{3}$ w.l.o.g. which yields $R_{\Omega}$ and $R_{\Pi}$, respectively. Then $R_{\Pi}$ splits up and can only vanish w.c. for $P[6]=0$ or $Q[14]$. It can easily be seen that the coefficients of the quadratic homogeneous polynomial $P=0$ in the unknowns $e_{1}, e_{2}$ cannot vanish w.c.. Therefore we set $Q=0$ which is also a quadratic polynomial in the unknowns $e_{1}, e_{2}$. We denote the coefficients of $e_{1}^{i} e_{2}^{j}$ of $Q$ by $Q_{i j}$. Now $Q_{02}=0$ implies $A_{5}=-\bar{X}_{2} a_{2} / \bar{x}_{5}$. Then we get $\bar{x}_{5}=-1 / \bar{X}_{2}$ from $Q_{11}=0$. Then $Q$ is fulfilled identically. Now $\Pi_{1000}=0$ cannot vanish w.c..

b. $\bar{X}_{2}=\bar{x}_{5}$ : In this case $\Pi_{0013}$ can only vanish w.c. for:

i. $A_{5}=a_{2}$ : Now $\Pi_{1000}$ is a factor of $\Pi$. Therefore we can only compute the resultant of $\Pi_{1000}$ and $\Omega$ with respect to $e_{3}$ w.l.o.g. which yields a homogeneous polynomial $R_{\Omega}[674]$ of degree 6 in $e_{0}, e_{1}, e_{2}$. It is again not difficult to verify that the coefficients of $R_{\Omega}$ cannot vanish w.c. (proof is left to the reader).

ii. $\bar{x}_{5}= \pm i, A_{5} \neq a_{2}$. Now it can easily be seen that $\Pi_{0002}$ cannot vanish w.c.. Therefore we can compute the resultant of $\Pi_{1000}$ and $\Omega$ resp. $\Pi$ 
with respect to $e_{3}$ w.l.o.g. which yields $R_{\Omega}$ and $R_{\Pi}$, respectively. Then $R_{\Pi}$ splits up and can only vanish w.c. for $P[5]=0$. It can easily be seen that the coefficients of the linear homogeneous polynomial $P=0$ in the unknowns $e_{1}, e_{2}$ cannot vanish w.c..

3. $\Omega_{0002}=0, \Omega_{0001} \Pi_{0003} \neq 0$ : W.l.o.g. we can express $L_{3}$ from $\Omega_{0002}=0$. Now the remaining discussion of this case can exactly be done as in item 1 of part [G1].

4. $\Omega_{0002}=\Omega_{0001}=0, \Pi_{0003} \neq 0$ : W.l.o.g. we can express $L_{3}$ from $\Omega_{0002}=0$ and $a_{2}$ from $\Omega_{1001}=0$. Then we compute the resultant of $\Pi_{1000}$ and $\Pi$ with respect to $e_{3}$ w.l.o.g. which yields $R_{\Pi}$. Now $R_{\Pi}$ splits up and can only vanish w.c. for $P[11]=0$ or $Q[49]=0$.

a. $P[11]=0$ : Now the coefficients of this equation can only vanish w.c. for:

$$
b_{2}=-B_{5}, \quad A_{5}=\bar{X}_{2} B_{5}, \quad a_{3}=B_{5}\left(\bar{X}_{2}-\bar{x}_{5}\right)+\bar{X}_{3} b_{3} .
$$

But then $\Pi_{1000}$ equals $e_{1} e_{3}^{2} B_{5}\left(\bar{X}_{2}-\bar{x}_{5}\right)$ which yields the contradiction.

b. $Q[49]=0$ : This is a quartic polynomial in the unknowns $e_{1}, e_{2}$. We denote the coefficients of $e_{1}^{i} e_{2}^{j}$ of $Q$ by $Q_{i j}$. Then $Q_{04}$ can only vanish w.c. for:

i. $b_{2}=-B_{5}: Q_{40}=0$ implies an expression for $a_{3}$. $Q_{31}$ cannot vanish w.c..

ii. $A_{5}=a_{3}+B_{5} \bar{x}_{5}-\bar{X}_{3} b_{3}$ : Then $Q_{40}$ can vanish w.c. for:

$\star b_{2}=B_{5}$ : Then $Q_{31}=0$ implies an expression for $a_{3}$. Finally $Q_{13}$ cannot vanish w.c..

$\star a_{3}=\bar{X}_{3} b_{3}$ : Now $Q_{31}$ can only vanish w.c. for $\bar{x}_{5}= \pm \bar{X}_{2}$. As for $\bar{x}_{5}=$ $-\bar{X}_{2}$ the expression $Q_{22}$ cannot vanish w.c. we set $\bar{x}_{5}=\bar{X}_{2}$. Then $Q$ is fulfilled identically. As now the coefficient of the highest exponent of $e_{2}$ in $\Pi_{1000}$ and $\Omega=0$ cannot vanish w.c. we can compute the resultant of $\Pi_{1000}=0$ and $\Omega$ with respect to $e_{2}$ w.l.o.g. which yields $R_{\Omega}$ [87]. Now it is not difficult to verify that the coefficients of $R_{\Omega}$ cannot vanish w.c. (proof is left to the reader).

5. $\Omega_{0002}=\Pi_{0003}=0, \Omega_{0001} \neq 0$ : We can express $L_{3}$ from $\Omega_{0002}=0$ and $\Pi_{0103}=0$ implies $b_{2}=\bar{x}_{5} A_{5}+B_{5}-\bar{X}_{2} a_{2}$.

a. $\bar{X}_{2} \neq \bar{x}_{5}$ : Under this assumption we can express $B_{5}$ from $\Pi_{0013}=0$. As $\Pi_{0002}$ cannot vanish w.c. we can compute the resultant of $\Pi_{1000}$ and $\Pi$ with respect to $e_{3}$, which yields $R_{\Pi}$. Now $R_{\Pi}$ splits up and can only vanish w.c. for $P[6]=0$ or $Q[14]=0$. As it can easily be seen, that the coefficients of $P[6]=0$ cannot vanish w.c., we set $Q[14]$ equal to zero, which is a quadratic polynomial in the unknowns $e_{1}, e_{2}$. We denote the coefficients of $e_{1}^{i} e_{2}^{j}$ of $Q$ by $Q_{i j}$. Then $Q_{02}=0$ implies $a_{2}=-\bar{x}_{5} A_{5} / \bar{X}_{2}$. Now $Q_{11}=0$ can only vanish w.c. for:

i. $\bar{X}_{2}=-\bar{x}_{5}$ : Then $Q_{20}$ can only vanish for $x_{5}= \pm 1$. In both cases $\Pi_{1000}=0$ yields the contradiction.

ii. $\bar{x}_{5}=-1 / \bar{X}_{2}, \bar{X}_{2}+\bar{x}_{5} \neq 0$ : Again, $\Pi_{1000}=0$ yields the contradiction.

b. $\bar{X}_{2}=\bar{x}_{5}$ : Now $\Pi_{0013}$ can only vanish w.c. for:

i. $a_{2}=A_{5}$ : Now $\Pi_{1000}$ is a factor of $\Pi$. Therefore we compute the resultant of $\Pi_{1000}$ and $\Omega$ with respect to $e_{3}$, which yields $R_{\Omega}$ [244]. Moreover, 
$R_{\Omega}=0$ is a homogeneous equation of degree 5 in $e_{0}, e_{1}, e_{2}$. Now it is not difficult to verify that the coefficients of $R_{\Omega}$ cannot vanish w.c. (proof is left to the reader).

ii. $x_{5}= \pm i, a_{2} \neq A_{5}$. In this case we can compute the resultant of $\Omega$ and the only non-contradicting factor of $\Pi_{1000}$ with respect to $e_{3}$, which yields $R_{\Omega}$ [207]. Moreover, $R_{\Omega}=0$ is a homogeneous equation of degree 4 in $e_{0}, e_{1}, e_{2}$. Then the coefficient of $e_{2}^{3}$ of $R_{\Omega}$ yields the contradiction.

6. $\Omega_{0002}=\Omega_{0001}=\Pi_{0003}=0$ : We can express $L_{3}$ from $\Omega_{0002}=0$ and $\Pi_{0001}=$ 0 implies $a_{2}=a_{3}+\bar{X}_{2} b_{2}-\bar{X}_{3} b_{3}$. Moreover we get $a_{3}=A_{5} \bar{X}_{3} b_{3}-\bar{x}_{5} B_{5}$ from $\Pi_{0013}=0$.

a. $\bar{X}_{2} \neq \bar{x}_{5}$ : Under this assumption we can express $A_{5}$ from $\Pi_{0103}=0$. As $\Pi_{0002}$ cannot vanish w.c. we can compute the resultant of $\Pi_{1000}$ and $\Pi$ with respect to $e_{3}$, which yields $R_{\Pi}$. Now $R_{\Pi}$ splits up and can only vanish w.c. for $P[6]=0$ or $Q[14]=0$. As it can easily be seen, that the coefficients of $P[6]=0$ cannot vanish w.c., we set $Q[14]$ equal to zero, which is a quadratic polynomial in the unknowns $e_{1}, e_{2}$. We denote the coefficients of $e_{1}^{i} e_{2}^{j}$ of $Q$ by $Q_{i j}$. Then $Q_{20}=0$ implies $B_{5}=-b_{2}$. Now $Q_{02}=0$ can only vanish w.c. for:

i. $\bar{X}_{2}=-\bar{x}_{5}$ : Then $Q_{11}=0$ yields the contradiction.

ii. $\bar{x}_{5}=-1 / \bar{X}_{2}, \bar{X}_{2}+\bar{x}_{5} \neq 0$ : Now $\Pi_{1000}=0$ yields the contradiction.

b. $\bar{X}_{2}=\bar{x}_{5}$ : Now $\Pi_{0103}$ can only vanish w.c. for:

i. $b_{2}=B_{5}$ : Now $\Pi_{1000}$ is a factor of $\Pi$. Moreover, $\Omega_{0020}$ cannot vanish w.c..

$\star A_{5} \neq 0$ : Under this assumption we can compute the resultant of $\Pi_{1000}$ and $\Omega$ with respect to $e_{2}$, which yields $R_{\Omega}$ [792]. The coefficient of $e_{0}^{6}$ of $R_{\Omega}$ cannot vanish w.c..

$\star A_{5}=0$ : Now the coefficient of $e_{2}^{2}$ of $\Pi_{1000}$ cannot vanish w.c., and therefore we can compute the resultant of $\Pi_{1000}$ and $\Omega$ with respect to $e_{2}$, which yields $R_{\Omega}[80]$. Then the coefficient of $e_{0}^{4}$ of $R_{\Omega}$ yields the contradiction.

ii. $X_{2}= \pm i, b_{2} \neq B_{5}$ : We distinguish two cases:

$\star 2 A_{5} \pm b_{2} i \mp B_{5} i \neq 0$ : Under this assumption the highest exponent of $e_{2}$ in $\Pi$ and $\Pi_{1000}$ cannot vanish w.c.. Therefore we can compute the resultant of the only non-contradicting factors of $\Pi_{1000}$ and $\Pi$ with respect to $e_{2}$, which yields $R_{\Pi}$. It can immediately be seen, that $R_{\Pi}$ cannot vanish w.c..

$\star A_{5}=\left( \pm B_{5} i \mp b_{2} i\right) / 2$ : Now $\Pi_{1000}$ can only vanish w.c. for $e_{1}=\mp e_{3}^{2} i / e_{2}$. Then it can immediately be seen, that $\Pi=0$ yields the contradiction.

Part [G2] $\Pi_{1000}=0$ vanishes identically for all $e_{1}, e_{2}, e_{3}$ : Now $\Pi_{1210}=0$ implies $b_{2}=-B_{5}$ and from $\Pi_{1012}=0$ we get $a_{2}=-A_{5} \bar{x}_{5} / \bar{X}_{2}$. Then $\Pi_{1120}=0$ and $\Pi_{1102}=$ 0 can only vanish w.c. for $\bar{X}_{2}=\bar{x}_{5}$. Now we distinguish the following cases:

1. $\Omega_{0200} \neq 0$ : As $\Pi_{0300}$ cannot vanish w.c. and due to our assumption $\Omega_{0200} \neq 0$ we can compute the resultant of $\Omega$ and $\Pi$ with respect to $e_{1}$ which yields a homogeneous polynomial $\Gamma[3200]$ of degree 6 in $e_{0}, e_{2}, e_{3}$. In the following we denote the coefficients of $e_{0}^{i}, e_{2}^{j}, e_{3}^{k}$ of $\Gamma$ by $\Gamma_{i j k}$. 
W.l.o.g. we can solve $\Gamma_{600}=0$ for $L_{3}$. Then $\Gamma_{303}=0$ implies $a_{3}=\bar{X}_{3} b_{3}+B_{5} \bar{x}_{5}-$ $A_{5}$. Now $\Gamma_{006}$ can only vanish w.c. for $\bar{X}_{5}\left(A_{5} B_{5}-\bar{X}_{3} B_{5} b_{3}-B_{5}^{2} \bar{x}_{5}\right)+A_{5} \bar{x}_{5} b_{3}=0$. Now we have to distinguish again two cases:

a. $\bar{X}_{3} B_{5}+\bar{x}_{5} b_{3} \neq 0$ : Under this assumption we can compute $A_{5}$ from $N=0$.

i. $\bar{X}_{3}-2 \bar{X}_{3}^{2} \bar{x}_{5}-\bar{x}_{5} \neq 0$ : Now we can solve the only non-contradicting factor of $\Gamma_{024}=0$ for $b_{3}$. Then $\Gamma_{042}=0$ yields the contradiction.

ii. $\bar{X}_{3}-2 \bar{X}_{3}^{2} \bar{x}_{5}-\bar{x}_{5}=0$ : W.l.o.g. we can solve this expression for $\bar{x}_{5}$. Now $\Gamma_{024}=0$ yields the contradiction.

b. $\bar{X}_{3}=-\bar{x}_{5} b_{3} / B_{5}$ : Now $N=0$ implies $B_{5}=b_{3}$. Then $\Gamma_{024}$ can only vanish w.c. for $b_{3}=-A_{5}\left(1+2 \bar{x}_{5}^{2}\right) / \bar{x}_{5}$. Finally $\Gamma_{060}=0$ yields the contradiction.

2. $\Omega_{0200}=0, \Omega_{0100} \neq 0$ : W.l.o.g. we can express $L_{3}$ from $\Omega_{0200}=0$. Then we solve $\Omega$ for $e_{1}$ and plug it into $\Pi$ which yields in the numerator a homogeneous polynomial $\Gamma[621]$ of degree 6 in $e_{0}, e_{2}, e_{3}$. Then the coefficient of $e_{0}^{6}$ of $\Gamma$ already yields the contradiction.

3. $\Omega_{0200}=\Omega_{0100}=0, \Pi_{0030} \neq 0$ : W.l.o.g. we can express $L_{3}$ from $\Omega_{0200}=0$ and $a_{3}$ from $\Omega_{0110}=0$. Now it can easily be seen that $\Omega_{0020}$ cannot vanish w.c.. Due to this fact an the assumption $\Pi_{0020} \neq 0$ we can compute the resultant of $\Omega$ and $\Pi$ with respect to $e_{2}$ which yields a homogeneous polynomial $\Gamma[838]$ of degree 6 in $e_{0}, e_{1}, e_{3}$. Again we get the contradiction from the coefficient of $e_{0}^{6}$ of $\Gamma$.

4. $\Omega_{0200}=\Omega_{0100}=\Pi_{0030}=0$ : W.l.o.g. we can express $L_{3}$ from $\Omega_{0200}=0$ and $a_{3}$ from $\Omega_{0110}=0$. Now $\Pi_{0030}=0$ implies $A_{5}=B_{5} \bar{x}_{5}$.

Now it can easily be seen that $\Omega_{0020}$ as well as $\Pi_{0020}$ cannot vanish w.c.. Therefore we can compute the resultant of $\Omega$ and $\Pi$ with respect to $e_{2}$ w.l.o.g. which yields a homogeneous polynomial $\Gamma[100]$ of degree 4 in $e_{0}, e_{1}, e_{3}$. Finally we get the contradiction from the coefficient of $e_{0}^{4}$ of $\Gamma$.

Due to the structure ${ }^{3}$ of $\Omega$ it can easily be seen, that $\Omega$ and $\Pi$ can only have a common factor, which does not depend on $e_{0}$ (cf. footnote 2) if $\Omega=0$ has this property too. As this case was already treated in part $[\mathrm{F}]$ we remain with the discussion of those cases excluded by the assumption $e_{0} e_{2}-e_{1} e_{3} \neq 0$ (cf. footnote 1 ).

\section{Proof for the case $e_{0} e_{2}-e_{1} e_{3}=0$}

We split up this section of the proof into three parts.

Part [A] As $e_{0}=e_{1}=e_{2}=e_{3}=0$ does not correspond with an Euclidean motion, we start by discussing the following 4 cases:

$$
e_{0}=e_{1}=e_{2}=0, \quad e_{0}=e_{1}=e_{3}=0, \quad e_{0}=e_{2}=e_{3}=0, \quad e_{1}=e_{2}=e_{3}=0 .
$$

We only discuss the case $e_{0}=e_{1}=e_{2}=0$ in more detail because the other 3 cases can be done analogously. Now $\Psi=0$ implies $f_{3}=0$. Then $\Omega_{1}=0$ yields an expression for $f_{2}$ and from $\Omega_{2}=0$ we get an expression for $f_{1}$. This cannot yield a 2-parametric self-motion as only the homogeneous parameters $e_{3}$ and $f_{0}$ are free.

$\overline{{ }^{3} \Omega: \sum_{i=0}^{3} c_{i} e_{i}^{2}+c_{4} e_{0} e_{3}+c_{5} e_{1}} e_{2}$ where $c_{0}, \ldots, c_{5}$ only depend on the geometry of the SG platform. 
Part [B] In this part we discuss the following four special cases:

1. $e_{0}=e_{1}=0$ : Due to part [A] we can assume w.l.o.g. $e_{2} e_{3} \neq 0$. We can compute $f_{2}$ w.l.o.g. from $\Psi=0$. Then $\Omega_{1}$ implies $f_{3}=-L_{1} e_{2} / 2$. Then $\Pi_{4}$ can only vanish w.c. for $g_{4}=-L_{1}$. Moreover, we can express $f_{1}$ from $\Pi_{5}$ w.l.o.g.. Finally the coefficients of $e_{2} f_{0}$ of $\Omega_{2}$ and $\Omega_{3}$ cannot vanish w.c..

2. $e_{2}=e_{3}=0$ : This case can be done analogously to the last one.

3. $e_{0}=e_{3}=0$ : Due to part [A] we can assume $e_{1} e_{2} \neq 0$. We can compute $f_{1}$ from $\Psi=0$. Then we can express $f_{0}$ from $\Pi_{4}=0$. Moreover, we can compute $f_{3}$ from $\Pi_{5}=0$. Now $\Omega_{1}, \Omega_{2}$ and $\Omega_{3}$ have to vanish independently of the choice of the unknowns $e_{1}, e_{2}, f_{2}$.

The coefficient of $e_{1}^{4}$ of $\Omega_{2}$ implies an expression for $h_{5}$. Then we get $L_{2}$ from the coefficient of $e_{1}^{1} e_{2}^{3}$ of $\Omega_{2}$ and $L_{3}$ from the coefficient of $e_{1}^{4}$ of $\Omega_{3}$. Then the coefficients of $e_{1}^{4}$ and $e_{2}^{4}$ of $\Omega_{1}$ imply $L_{1}=g_{4}=0$. Now we can compute $a_{2}$ from the coefficient of $e_{1}^{1} e_{2}^{3}$ of $\Omega_{1}$. Moreover, the coefficient of $e_{1}^{3} e_{2}^{1}$ of $\Omega_{1}$ implies $B_{5}=\bar{x}_{5} A_{5}$ and from the coefficient of $e_{1}^{1} e_{2}^{3}$ of $\Omega_{3}$ we get $a_{3}=A_{5}\left(1+\bar{x}_{5}^{2}\right)-\bar{X}_{3} b_{3}$. Then the coefficient of $e_{1}^{2} e_{2}^{2}$ of $\Omega_{1}$ can only vanish w.c. for $x_{5}=\mp i$. Then the coefficient of $e_{2}^{4}$ of $\Omega_{2}$ implies $X_{2}= \pm i$. Finally, the coefficient of $e_{2}^{4}$ of $\Omega_{3}$ yields the contradiction.

4. $e_{1}=e_{2}=0$ : This case can be done analogously to the last one.

Part [C] Due to the discussion of the special cases in part [A] and part [B] we can assume w.l.o.g. $e_{0} e_{1} e_{2} e_{3} \neq 0$. Therefore we can solve $e_{0} e_{2}-e_{1} e_{3}=0$ w.l.o.g. for $e_{2}$. Moreover, we can solve $\Psi, \Omega_{1}, \Pi_{4}, \Pi_{5}$ w.l.o.g. for $f_{0}, f_{1}, f_{2}, f_{3}$.

Now $\Omega_{2}$ and $\Omega_{3}$ have to vanish independently of the choice of the unknowns $e_{0}, e_{1}, e_{3}$. Therefore the coefficient of $e_{0}^{6}$ of $\Omega_{2}$ implies $L_{1}=g_{4}$. Then the coefficient of $e_{0}^{5} e_{3}$ of $\Omega_{2}$ yields an expression for $L_{2}$. Now we get $g_{4}=2 a_{2}-2 \bar{X}_{2} b_{2}$ from the coefficient of $e_{0}^{4} e_{3}^{2}$ of $\Omega_{2}$. Moreover, we get $a_{2}=\bar{X}_{2} b_{2}$ from the coefficient of $e_{1}^{2} e_{3}^{4}$ of $\Omega_{2}$. Finally the coefficient of $e_{0} e_{1}^{2} e_{3}^{3}$ of $\Omega_{2}$ cannot vanish w.c..

This finishes the proof of Theorem 2 .

\section{Conclusion and future research}

In this paper we presented the basic result (cf. Theorem 2) on type II Darboux Mannheim (DM) self-motions of planar SG platforms. Due to Lemma 2 of [6] and Theorem 1 we can replace the word "or" in Theorem 2 by the word "and"; i.e. with exception of the two special cases there always exist three collinear platform points $\mathrm{u}_{i}, \mathrm{u}_{j}, \mathrm{u}_{k}$ and three collinear base points $\mathrm{U}_{l}, \mathrm{U}_{m}, \mathrm{U}_{n}$ beside the points $\mathrm{U}_{1}, \mathrm{U}_{2}, \mathrm{U}_{3}$ and $\mathrm{u}_{4}, \mathrm{u}_{5}, \mathrm{u}_{6}$ where $(i, j, k, l, m, n)$ consists of all indices from 1 to 6 .

The presented basic result raises the hope of giving a complete classification of type II DM self-motions in the future, which would be an important step in solving the famous Borel Bricard problem. On base of Theorem 2 the work towards this goal is in progress. 
Acknowledgements This research is supported by Grant No. I 408-N13 of the Austrian Science Fund FWF within the project "Flexible polyhedra and frameworks in different spaces", an international cooperation between FWF and RFBR, the Russian Foundation for Basic Research.

\section{References}

1. Borel, E.: Mémoire sur les déplacements à trajectoires sphériques, Mém. présenteés par divers savants, Paris(2), 33, 1-128 (1908).

2. Borras, J., Thomas, F., Torras, C.: Singularity-invariant leg rearrangements in doubly-planar Stewart-Gough platforms, In Proc. of Robotics Science and Systems, Zaragoza, Spain (2010).

3. Bricard, R.: Mémoire sur les déplacements à trajectoires sphériques, Journ. École Polyt.(2), 11, 1-96 (1906).

4. Husty, M.: E. Borel's and R. Bricard's Papers on Displacements with Spherical Paths and their Relevance to Self-Motions of Parallel Manipulators, Int. Symp. on History of Machines and Mechanisms (M. Ceccarelli ed.), 163-172, Kluwer (2000).

5. Husty, M., Mielczarek, S., Hiller, M.: A redundant spatial Stewart-Gough platform with a maximal forward kinematics solution set, Advances in Robot Kinematics: Theory and Applications (J. Lenarcic, F. Thomas eds.), 147-154, Kluwer (2002).

6. Karger, A.: Architecture singular planar parallel manipulators, Mechanism and Machine Theory 38 (11) 1149-1164 (2003).

7. Karger, A.: New Self-Motions of Parallel Manipulators, Advances in Robot Kinematics: Analysis and Design (J. Lenarcic, P. Wenger eds.), 275-282, Springer (2008).

8. Karger, A.: Self-motions of Stewart-Gough platforms, Computer Aided Geometric Design, Special Issue: Classical Techniques for Applied Geometry (B. Jüttler, O. Röschel, E. Zagar eds.) 25 (9) 775-783 (2008).

9. Mielczarek, S., Husty, M.L., Hiller, M.: Designing a redundant Stewart-Gough platform with a maximal forward kinematics solution set, In Proc. of the International Symposion of Multibody Simulation and Mechatronics (MUSME), Mexico City, Mexico, September 2002.

10. Nawratil, G.: Types of self-motions of planar Stewart Gough platforms, under review.

11. Vogler, H.: Bemerkungen zu einem Satz von W. Blaschke und zur Methode von Borel-Bricard, Grazer Mathematische Berichte 352 1-16 (2008). 